\title{
The long non-coding RNA HOTAIRM1 promotes tumor aggressiveness and radiotherapy resistance in glioblastoma
}

\author{
Ulvi Ahmadov ${ }^{1,2,3,4,15}$, Daniel Picard ${ }^{1,2,3,4,15}$, Jasmin Bartl ${ }^{1,2,3,4}$, Manuela Silginer ${ }^{5}$, Marija Trajkovic-Arsic ${ }^{6,7}$, Nan Qin ${ }^{1,2,3,4}$, \\ Lena Blümel ${ }^{1,2,3,4}$, Marietta Wolter ${ }^{4}$, Jonathan K. M. Lim ${ }^{4}$, David Pauck ${ }^{1,2,3,4}$, Alina Marie Winkelkotte ${ }^{6,7}$, Marlen Melcher ${ }^{8}$, \\ Maike Langini ${ }^{9,10}{ }^{9,}$ Viktoria Marquardt ${ }^{1,2,3,4}$, Felix Sander ${ }^{1,2,3,4}$, Anja Stefanski ${ }^{9,10}$, Sascha Steltgens ${ }^{4}$, Christina Hassiepen ${ }^{11}$, \\ Anna Kaufhold 1,2,3,4 , Frauke-Dorothee Meyer ${ }^{1,2,3,4}$, Annette Seibt ${ }^{8}$, Lara Kleinesudeik ${ }^{12,13}$, Anika Hain ${ }^{14}$, Carsten Münk ${ }^{14}$, \\ Christiane Brigitte Knobbe-Thomsen ${ }^{4}$, Alexander Schramm ${ }^{11}$, Ute Fischer ${ }^{3}$, Gabriel Leprivier (iD ${ }^{4}$, Kai Stühler ${ }^{9,10}$, Simone Fulda (iD ${ }^{12,13}$ \\ Jens T. Siveke ${ }^{6,7}$, Felix Distelmaier ${ }^{8}$, Arndt Borkhardt (iD ${ }^{1,2,3}$, Michael Weller ${ }^{5}{ }^{5}$, Patrick Roth ${ }^{5}$, Guido Reifenberger ${ }^{1,2,4,16}$ and
} Marc Remke $e^{1,2,3,4,16 \bowtie}$

(c) The Author(s) 2021

\begin{abstract}
Glioblastoma is the most common malignant primary brain tumor. To date, clinically relevant biomarkers are restricted to isocitrate dehydrogenase (IDH) gene 1 or 2 mutations and O6-methylguanine DNA methyltransferase (MGMT) promoter methylation. Long non-coding RNAs (IncRNAs) have been shown to contribute to glioblastoma pathogenesis and could potentially serve as novel biomarkers. The clinical significance of HOXA Transcript Antisense RNA, Myeloid-Specific 1 (HOTAIRM1) was determined by analyzing HOTAIRM1 in multiple glioblastoma gene expression data sets for associations with prognosis, as well as, IDH mutation and MGMT promoter methylation status. Finally, the role of HOTAIRM1 in glioblastoma biology and radiotherapy resistance was characterized in vitro and in vivo. We identified HOTAIRM1 as a candidate IncRNA whose up-regulation is significantly associated with shorter survival of glioblastoma patients, independent from IDH mutation and MGMT promoter methylation. Glioblastoma cell line models uniformly showed reduced cell viability, decreased invasive growth and diminished colony formation capacity upon HOTAIRM1 down-regulation. Integrated proteogenomic analyses revealed impaired mitochondrial function and determination of reactive oxygen species (ROS) levels confirmed increased ROS levels upon HOTAIRM1 knock-down. HOTAIRM1 knock-down decreased expression of transglutaminase 2 (TGM2), a candidate protein implicated in mitochondrial function, and knock-down of TGM2 mimicked the phenotype of HOTAIRM1 down-regulation in glioblastoma cells. Moreover, HOTAIRM1 modulates radiosensitivity of glioblastoma cells both in vitro and in vivo. Our data support a role for HOTAIRM1 as a driver of biological aggressiveness, radioresistance and poor outcome in glioblastoma. Targeting HOTAIRM1 may be a promising new therapeutic approach.
\end{abstract}

Cell Death and Disease (2021)12:885; https://doi.org/10.1038/s41419-021-04146-0

\section{INTRODUCTION}

Glioblastoma is the most malignant type of astrocytic glioma and the most common malignant primary brain tumor [1]. According to the World Health Organization (WHO) classification of central nervous system tumors, glioblastomas correspond to WHO grade IV and are stratified based on their isocitrate dehydrogenase (IDH) 1 or 2 gene mutation status into two biologically and clinically distinct entities [2]. IDH-wildtype glioblastomas account for more than $90 \%$ of the tumors and preferentially manifest de novo with short clinical history in patients older than 50 years. In contrast, IDH-mutant glioblastomas, which have recently been re-named as astrocytoma, IDH-mutant, WHO grade 4 [3], are less common, typically occur in patients younger than 50 years and may develop from pre-

\footnotetext{
${ }^{1}$ Division of Pediatric Neuro-Oncogenomics, German Cancer Research Center (DKFZ), Heidelberg, Germany. ${ }^{2}$ German Consortium for Translational Cancer Research (DKTK), partner site Essen/Düsseldorf, Düsseldorf, Germany. ${ }^{3}$ Department of Pediatric Oncology, Hematology, and Clinical Immunology, Medical Faculty, University Hospital Düsseldorf, Düsseldorf, Germany. ${ }^{4}$ Department of Neuropathology, Medical Faculty, Heinrich Heine University, Düsseldorf, Germany. ${ }^{5}$ Department of Neurology, University Hospital and University of Zurich, Zurich, Switzerland. ${ }^{6}$ Bridge Institute of Experimental Tumor Therapy, West German Cancer Center, University Medicine Essen, Essen, Germany. ${ }^{7}$ Division of Solid Tumor Translational Oncology, German Cancer Research Center (DKFZ) and German Cancer Consortium (DKTK), partner site Essen, Heidelberg, Germany. ${ }^{8}$ Department of General Pediatrics, Neonatology and Pediatric Cardiology, University Hospital Düsseldorf, Düsseldorf, Germany. ${ }^{9}$ Institute for Molecular Medicine I, Medical Faculty, Heinrich

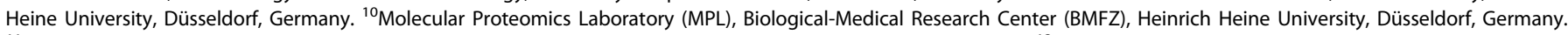
${ }^{11}$ Department of Molecular Oncology, West German Cancer Center, University Hospital Essen, Essen, Germany. ${ }^{12}$ Institute for Experimental Cancer Research in Pediatrics, Goethe University Frankfurt, Frankfurt, Germany. ${ }^{13}$ German Cancer Consortium (DKTK), Partner Site Frankfurt and German Cancer Research Center (DKFZ), Heidelberg, Germany.

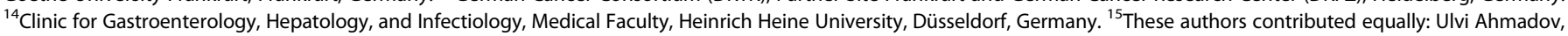
Daniel Picard. ${ }^{16}$ These authors jointly supervised this work: Guido Reifenberger, Marc Remke. ${ }^{凶}$ email: marc.remke@med.uni-duesseldorf.de Edited by Dr Giovanni Blandino
} 
existing IDH-mutant lower grade astrocytomas [2, 4]. Current standard therapy of glioblastoma consists of surgical resection followed by radiotherapy with concomitant and maintenance temozolomide (TMZ) chemotherapy [5]. Recently, tumor treatment fields (TTF) have been reported and approved as an additional treatment [6]. However, the outcome of IDH-wildtype glioblastoma patients remains poor, with median overall survival limited to $15-18$ months and reported five-year survival rate of less than 10\% [7]. The majority of glioblastomas demonstrate remarkable resistance to radiation and chemotherapy either upfront or during the course of treatment $[8,9]$. Only a few alterations have been established as clinically relevant biomarkers to date, including IDH mutation as a diagnostic and prognostic biomarker, as well as aberrant promoter methylation of the O6-methylguanine DNA methyltransferase (MGMT) gene as a predictor of benefit from TMZ [10-12]. However, clinically active molecularly targeted therapy approaches are still missing [13].

To further elucidate the molecular pathogenesis of glioblastoma, we evaluated long non-coding RNA (IncRNA) expression profiles in microarray-based gene expression data sets. LncRNAs are non-coding transcripts that are longer than 200 nucleotides [14]. Recent reports have indicated important roles of aberrant IncRNA expression in tumorigenesis, progression and therapy resistance of various cancers [15-17]. We identified the IncRNA HOXA transcript antisense RNA myeloid-specific 1 (HOTAIRM1) as a candidate IncRNA which maps within the HOXA gene cluster on the short arm of chromosome 7 and was originally implicated in myelopoiesis through modulation of gene expression in the HOXA cluster [18]. Previous studies have reported that HOTAIRM1 expression is increased in high-grade gliomas and in recurrent compared to primary glioblastomas $[19,20]$. Recently, HOTAIRM1 has been shown to promote glioma growth and invasion through up-regulation of HOXA1 expression [21], through longrange chromatin interactions within HOXA cluster genes [22] and regulating SNAI2 [23]. Moreover, HOTAIRM1 has been proposed to promote glioma growth by acting as a sponge for several tumor suppressive miRNAs [24-26]. Here, we extend these findings by providing further clinical and functional evidence implicating HOTAIRM1 as a driver of tumor aggressiveness that contributes to radioresistance and poor outcome of glioblastoma patients.

\section{MATERIAL AND METHODS \\ Cell culture}

The glioblastoma cell lines T98G, U251MG and U87MG were obtained from American Type Culture Collection (ATCC, Manassas, VA, USA). LN-18 and LN-229 cell lines were provided by Dr. M. Hegi, Lausanne, while the SF126 cell line was obtained from the Japanese Collection of Research Bioresources Cell Bank (JCRB, Osaka, Japan). All lines were authenticated by short tandem repeat (STR) profiling and tested for mycoplasma contamination. Cell lines were grown in Dulbecco's modified Eagle's medium (\#31966-021, DMEM, Thermo Fischer Scientific, Waltham, MA, USA) supplemented with $10 \%$ heat-inactivated fetal bovine serum (FBS, \#F9665, Sigma-Aldrich, St. Louis, MO, USA) and 1\% penicillin-streptomycin (\#P4333, Sigma-Aldrich) in a humidified atmosphere with $5 \% \mathrm{CO}_{2}$ atmosphere at $37^{\circ} \mathrm{C}$. Stable cells were grown and/or selected with the culture media containing either blasticidin [T98G, LN-18, SF126 and U87MG] $(20 \mu \mathrm{g} / \mathrm{ml}$, \#ant-bl-1, InvivoGen, San Diego, CA, USA) or puromycin [LN-229and U251MG] (2 $\mu \mathrm{g} / \mathrm{ml}$, \#ant-pr-5, InvivoGen).

\section{RNA preparation and real-time quantitative PCR}

Total RNA extraction was performed by using TRIzol (\#15596026, Thermo Fischer Scientific) or by using a Maxwell RSC instrument (\#AS1340, RSC simplyRNA Tissue, Promega, Madison, WI, USA). Reverse transcription of total RNA ( $1 \mu \mathrm{g}$ ) was carried out using the MMLV-RT kit (\#M3683, Promega) with random hexamers. Real-time quantitative PCR (RT-qPCR) was subsequently performed with a 1:10 dilution of reverse-transcribed CDNA using a CFX384 Touch $^{\text {TM }}$ Real-Time PCR Detection System (Bio-Rad, Hercules, CA, USA). RT-qPCR TagMan Universal Master Mix II (\#4440038, Thermo Fischer Scientific) was employed, with TagMan probes specific for HOTAIRM1- (exon 1-3, \#Hs.PT.58.45434173, Integrated DNA Technologies (IDT), Coralville, IA, USA), TGM2 (\#Hs.PT.58.23141755, IDT) or PGK1 (\#Hs. PT.58 v.606641, IDT). RT-qPCR results were evaluated by $2^{-\Delta \Delta C t}$ method [27] using PGK1 expression levels as a housekeeping gene control.

\section{Generation of HOTAIRM1 or TGM2 knock-down glioblastoma} cells

For transient knock-down of HOTAIRM1 and TGM2,T98G, LN-229, LN-18, SF126 or U251 glioblastoma cells were seeded $(100,000$ cells per well) into 6-well plates the day before knock-down. Specific knock-down of HOTAIRM1 or TGM2 and the corresponding non-target negative controls was achieved by using HOTAIRM1-specific siPOOLs (\#100506311, siTOOLs Technology, Planegg, Martinsried, Germany), TGM2-specific siPOOLs (\#7052 - TGM2 (human), siTOOLs Technology) and non-target siPOOLs (Neg. control siPOOL $5 \mathrm{nmol}$, siPOOL Technology), respectively. Transfection of siPOOLs was performed using Lipofectamine RNAiMAX Transfection Reagent (\#13778150, Thermo Fischer Scientific). Briefly, 7.5 $\mu$ l RNAiMAX Transfection Reagent was diluted in $125 \mu$ l Opti-MEM (\#31985062, Thermo Fischer Scientific) pipetted into a well with $0.5 \mu \mathrm{l}(5 \mathrm{pM})$ siPOOL in $125 \mu \mathrm{l}$ Opti-MEM.

Lentivirus-containing HOTAIRM1 shRNA constructs (sequence is $5^{\prime}$ GGAGACTGGTAGCTTATTAAA-3') and non-target negative control shRNA constructs (sequence is $5^{\prime}$-CCTAAGGTTAAGTCGCCCTCG-3') were obtained from IDT and the sequences were obtain from a previous study [28]. HOTAIRM1-pLK0.1-TRC plasmid and third generation lentiviral packing plasmids (pMDL/pRRE, pRSV-Rev and pMD2.G) were transfected into HEK293 T cells using polyethylenimine (\#408727, PEl, Sigma-Aldrich). Fresh culture media was added (without antibiotics) after 24 and $48 \mathrm{~h}$ post transfection. The virus-containing medium ( 48 and $72 \mathrm{~h}$ after transfection) was stored at $-80^{\circ} \mathrm{C}$. Glioblastoma cells (LN-229, LN-18, SF126 and U87MG) were seeded in 6-well plates the day before transduction and were infected with $1.5 \mathrm{ml}$ of the viral suspension containing DMEM, $10 \%$ FBS and $2 \mu \mathrm{g} / \mathrm{ml}$ polybrene (\#107689, hexadimethrine bromide, SigmaAldrich) for stable cell line generation. The virus-containing medium was removed $24 \mathrm{~h}$ post transduction and replaced with medium containing puromycin or blasticidin for at least a week.

\section{Determination of cell viability}

Cells were seeded (1000-4000 cells per well) into white-bottom 96-well plates (\#136101, Thermo Fischer Scientific) and incubated for $72 \mathrm{~h}$. Afterwards, cells were incubated with sterile $100 \mu \mathrm{l}$ of 1:1 diluted (with PBS) CellTiter-Glo (\#G7570, Promega) for $10 \mathrm{~min}$ at RT, shaken for $2 \mathrm{~min}$ and the absorbance was measured with a Spark $10 \mathrm{M}$ microplate reader (Tecan, Maennedorf, Switzerland). All experiments were independently repeated at least three times.

\section{Determination of cell invasion in vitro}

Corning BioCoat ${ }^{T M}$ Matrigel Invasion Chambers (\#354480, Corning, Bedford, MA, USA) were used to determine invasive capacity of glioblastoma cells in vitro. Transwell membranes were activated by adding $500 \mu \mathrm{l}$ serum-free medium for $2 \mathrm{~h}$ at room temperature. Afterwards, $750 \mu \mathrm{l}$ medium containing $10 \%$ FBS was added into the lower chamber. $2.5 \times 10^{5}$ cells in $500 \mu l$ were resuspended in serum-free medium and were added to the upper chamber. The chambers were removed after $48 \mathrm{~h}$ incubation $\left(a t 37^{\circ} \mathrm{C}\right.$ ), the medium was removed and the membrane was washed once with PBS. Next, the cells that had migrated across the polycarbonate membrane were fixed with methanol for $2 \mathrm{~min}$ and stained with $1 \%$ toluidine blue for $2 \mathrm{~min}$. The membrane was washed 4 times with distilled water and cells remaining in the upper chamber were removed using a cotton swab. Membranes were allowed to air dry for a minimum of one hour before being mounted with vectashield mounting medium (\#H-1000, Vector laboratories, Burlingame, CA, USA) on a glass slide. Finally, six random fields were selected and imaged (20X) using an Axiovert 200 microscope (Zeiss, Oberkochen, Germany) and the AxioVision (Version 4.8) software. Invading cells were counted for each membrane. All experiments were independently repeated at least three times.

\section{Determination of colony formation and in vitro radiosensitivity}

Cells were harvested using trypsin, counted with Vi-CELL XR (Beckman Coulter, Brea, CA, USA), plated on culture-treated $100 \mathrm{~mm}$ dishes (500 - 1000 cells per plate) and cultured for 21 days. At the end of the incubation period, media was removed from dishes and cells were washed 
once in PBS and fixed with $10 \%$ formalin for $45 \mathrm{~min}$. Cells were then stained with $0.1 \%$ crystal violet for $1 \mathrm{~h}$, washed in $\mathrm{H}_{2} \mathrm{O}$ to remove excess dye and were allowed to air dry overnight. The following day, colonies that were visible to the naked eye were counted. All experiments were repeated at least three times.

For the in vitro radiation assay, cells were irradiated at 2 and $4 \mathrm{~Gy}$ using a Gulmay RS225 irradiation machine (Gulmay GmbH, Krefeld, Germany). Afterwards, the colony formation assay was performed as described above to evaluate the effect of the irradiation. For determing the efect of radiation on HOTAIRM 1 expression, 4Gy irradiated cells were seeded $(100,000$ cells per well) into 6-well plates and samples were harvested $48 \mathrm{~h}$ post-seeding. HOTAIRM1 transcript levels were measured using qRT-PCR and results were validated using published data GSE153982 and GSE111247 [29].

\section{miRNA-175-5p transient over expression}

For transient over-expression of miR-17-5p mimic, $2 u L$ of hsa-miR-17-5p mirVana mimic $(50 \mu \mathrm{M}$ concentration; \#4464066, Ambion) or mirVana

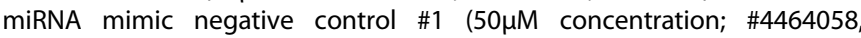
Ambion) was mixed with $2 \mathrm{uL}$ of Lipofectamine 2000 Transfection Reagent

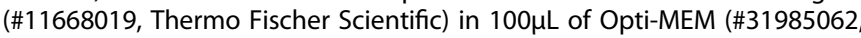
Thermo Fischer Scientific), and incubated for $15 \mathrm{~min}$.

Each transfection mix was placed into an individual well of a 6-well plate followed by addition of a $2 \mathrm{~mL}$ cell suspension of $\mathrm{LN}-229$ glioblastoma cells with a final seeding density of 150,000 cells per well. Transfections were harvested $72 \mathrm{~h}$ post-transfection.

\section{Luciferase reporter assay}

Plasmid insert (hg38, chr20:38,137,943-38,138,030), corresponding to the predicted miR-17-5p binding site within the $3^{\prime} U T R$ of TGM2, was subcloned into psiCHECK-2 vector (Promega, \# C8021). The miR-17-5p binding site 5'gtcctaagCACTTTataaa-3' was mutated to 5'- gtcctaagAAAAAAataaa-3'. The correctness of insert orientations was confirmed by sequencing. The reporter activity was measured by using Dual-Luciferase Reporter Assay System (Promega, \#E1910) according to the manufacturer's instructions. The 3'UTR- Luciferase reporter gene assays were performed as described previously (Wolter et al., 2016) except for transfecting cells with $50 \mathrm{nM}$ of miRNA mimic hsa-miR-17-5p (ThermoFisher Scientific, \# 4464066) and control cells with $50 \mathrm{nM}$ of the miRNA Negative Control \#1 (ThermoFisher Scientific, \# 4464058).

\section{In vivo mouse experiments}

All animal experiments were performed in accordance with the guidelines of Swiss federal law on animal protection. CD1 Foxn1 nude mice were purchased from Charles River Laboratories (Wilmington, MA, USA). Eleven 6-10-week-old mice per group were used in all experiments. Mice were anaesthetized using an intraperitoneal 3 component injection consisting of fentanyl, midazolam and medetomidin, fixed under a stereotactic device (Stoelting, Wood Dale, IL, USA) and a burr hole was drilled in the skull $2 \mathrm{~mm}$ lateral and $1 \mathrm{~mm}$ posterior to the bregma. A Hamilton syringe needle was introduced to a depth of $3 \mathrm{~mm}$ and LN-229 human glioma cells $(75,000)$ in a volume of $2 \mu \mathrm{l}$ phosphate-buffered saline (PBS) were injected into the right striatum. Local cranial radiotherapy with a single dose of 12 Gy was performed at day 15 after tumor implantation using a Gulmay $200 \mathrm{kV}$ X-ray unit at $1 \mathrm{~Gy} / \mathrm{min}$ at room temperature. The mice were observed daily and euthanized when neurological symptoms developed. No blinding was done for mouse experiments.

\section{Western blot analysis}

Cells were lysed and and total proteins were extracted using RIPA lysis buffer (\#20-188, Merck Millipore, Burlington, MA, USA) supplemented with protease and phosphatase inhibitor cocktail from Roche (\#04693132001 and \#04906837001, Sigma-Aldrich). Proteins were quantified with the Bradford method using the Protein Assay Dye Reagent (\#500-0006, BioRad). Samples were separated by SDS-PAGE and transferred to a nitrocellulose membrane (\#10600002, Sigma-Aldrich) by wet blot using the Mini Gel Tank and Blot Module (\#A25977 and \#B1000, Thermo Fischer Scientific). The membrane was incubated with rabbit anti-TGM2 (\#3557 S, D11A6, 1:1000, Cell Signaling, Danvers, MA, USA), and mouse anti-Actin B (\#MAB1501, clone 4, 1:5000, Merck Millipore) primary antibodies overnight at $4^{\circ} \mathrm{C}$. Next, the membrane incubated with species-specific, peroxidase- coupled secondary antibodies (anti-rabbit-HRP, \#7074 S, 1:5000, Cell Signaling or anti-mouse-HRP, \#H2014, 1:5000, Santa Cruz Biotechnology, Dallas, TX, USA) for an hour at RT. Finally, proteins were visualized using the SuperSignal West Femto Maximum Sensitivity Substrate (\#34095, Thermo Fischer Scientific) and detected using the LAS-3000 Imaging System (Fujifilm, Minato, Tokyo, Japan).

\section{RNA sequencing}

Total RNA was isolated from siRNA-mediated HOTAIRM1 knock-down and control cells of T98G, LN-229 and U251 glioblastoma cell lines $72 \mathrm{~h}$ post-transfection using TRIZol reagent. $500 \mathrm{ng}$ total RNA was processed using the TruSeq RNA Sample Preparation v2 kit (low-throughput protocol; Illumina, San Diego, CA, USA) to prepare the barcoded libraries. Libraries were validated and quantified using either DNA 1000 or highsensitivity chips on a Bioanalyzer (Agilent, Santa Clara, CA, USA). 7.5 pM denatured libraries were input into cBot (Illumina), followed by deep sequencing using HiSeq 2500 (Illumina) for 101 cycles, with an additional seven cycles for index reading. Fastq files were imported into Partek Flow (Partek Incorporated, St. Louis, MO, USA). Quality analysis and quality control were performed on all reads to assess read quality and to determine the amount of trimming required (both ends: 13 bases $5^{\prime}$ and 1 base $3^{\prime}$ ). Trimmed reads were aligned against the hg38 genome using the STAR v2.4.1d aligner. Unaligned reads were further processed using Bowtie 2 v2.2.5 aligner. Finally, aligned reads were combined before quantifying the expression against the ENSEMBL (release 84) database using the Partek Expectation-Maximization algorithm. Partek Flow default settings were used in all analyses. RNA sequencing data has been deposited in the NCBI GEO dataset repository under the identifyer GSE152147.

\section{Mass spectrometry-based proteome analyses}

For mass spectrometry (MS)-based proteome analyses, proteins were extracted from frozen cell pellets from siRNA-mediated HOTAIRM1 knockdown and control cells of the T98G, LN-229 and U251 cell lines. In addition, proteins were extracted from shRNA-mediated HOTAIRM1 knock-down and control cells of LN-229 cells as described. MS-based proteome analyses were performed as described before [30]. Cells were homogenized in urea buffer with a TissueLyser (Qiagen, Hilden, Germany) and subsequent sonication. After centrifugation for $15 \mathrm{~min}$ at $14,000 \mathrm{x} \mathrm{g}$ and $4{ }^{\circ} \mathrm{C}$, supernatants were collected. Protein concentration was determined via Pierce $660 \mathrm{~nm}$ Protein Assay (Thermo Fischer Scientific) and $10 \mu \mathrm{g}$ protein per sample were desalted through electrophoretic migration at $50 \mathrm{~V}$ for 10 min on a $4-12 \%$ Bis-Tris polyacrylamide gel (\#EC62352BOX, Novex NuPAGE, Thermo Fischer Scientific). After silver staining, protein bands were cut out, reduced, alkylated and digested with trypsin before peptide extraction via sonication. Peptides were dissolved and diluted with $0.1 \%$ TFA (v/v).

For MS-based proteome analyses, $15 \mu \mathrm{L}$ peptide solution per sample was analyzed on a nano-high-performance liquid chromatography electrospray ionization mass spectrometer. The analytical system was composed of an RSLCnano U3000 HPLC coupled to a QExactive Plus mass spectrometer via a nano-electrospray ion source (Thermo Fischer Scientific). Injected peptides were concentrated and desalted at a flow rate of $6 \mu \mathrm{L} / \mathrm{min}$ on a trapping column (Acclaim PepMao C18, $2 \mathrm{~cm} \times 100 \mu \mathrm{m} \times 3 \mu \mathrm{m}$ particle size, $100 \AA$ pore size, Thermo Fischer Scientific) with $0.1 \%$ TFA (v/v) for $10 \mathrm{~min}$. Subsequently, peptides were separated at a constant flowrate of $300 \mathrm{~nL} / \mathrm{min}$ over a 120 min gradient on an analytical column (Acclaim PepMap RSLC C18, $25 \mathrm{~cm} \times 75 \mu \mathrm{m} \times 2 \mu \mathrm{m}$ particle size, $100 \AA$ pore size, Thermo Fischer Scientific) at $60^{\circ} \mathrm{C}$. Separation was achieved through a gradient from 4 to $40 \%$ solvent $B$ (solvent A: $0.1 \%(\mathrm{v} / \mathrm{v})$ formic acid in water, solvent $\mathrm{B}: 0.1 \%(\mathrm{v} / \mathrm{v})$ formic acid, $84 \%(\mathrm{v} / \mathrm{v})$ acetonitrile in water). Afterwards, peptides were ionized at a voltage of $1,400 \mathrm{~V}$ and introduced into the mass spectrometer operated in positive mode. Mass spectrometry scans were recorded in profile mode in a range from $350-2000 \mathrm{~m} / \mathrm{z}$ at a resolution of 70,000 , while tandem mass spectra were recorded at a resolution of 17,500 . Tandem mass spectra were recorded with a data-dependent Top 10 method and $30 \%$ normalized collision energy. Dynamic exclusion was activated with a repeat count of 1 for $100 \mathrm{~s}$.

Proteome Discoverer (version 1.4.1.14, Thermo Fisher Scientific) was applied for peptide/protein identification with Mascot (version 2.4, Matrix 
Science, London, UK) as search engine employing the UniProt database (human; including isoforms; date 2016-03-01). A false discovery rate of $1 \%$ $(p \leq 0.01)$ on peptide level was set as identification threshold. Proteins were quantified with Progenesis QI for Proteomics (Version 2.0, Nonlinear Dynamics, Waters Corporation, Newcastle upon Tyne, UK). The mass spectrometry proteomics data has been deposited to the ProteomeXchange Consortium via the PRIDE [31] partner repository with the data set identifier PXD020141.

\section{Determination of superoxide levels}

Cellular and mitochondrial superoxide levels were determined by labeling cells with dihydroethidium (HEt, $10 \mu \mathrm{M}, 10 \mathrm{~min}$, and $37^{\circ} \mathrm{C}$; \#D11347, Thermo Fischer Scientific) or the mitochondria-targeted variant MitoSOX ${ }^{\mathrm{TM}}$ Red $\left(5 \mu \mathrm{M}, 10 \mathrm{~min}, 37^{\circ} \mathrm{C}\right.$; \#M36008, Thermo Fischer Scientific) as described elsewhere [32]. The staining reactions were stopped by washing the cells three times with PBS. The red fluorescence was documented using an Axio Observer Z1 microscope (Zeiss) with the dihydroethidium filter set (F39500, F48-515, F47-895; AHF Analysetechnik, Tuebingen, Germany). Images were analyzed and fluorescence intensity was quantified using ImageJ software (Wayne Rasband at the National Institutes of Health; http:// rsbweb.nih.gov/ij/).

\section{Bioinformatic and statistical analyses}

Statistical analyses were preformed by using GraphPad Prism (version 5.0) (https://www.graphpad.com/scientific-software/prism/). Experimental data are represented as mean \pm SEM based on at least three independent experiments. The two-way ANOVA test was used for statistical analyses of qRT-PCR, viability, invasion, colony formation and reactive oxygen species (ROS) staining assays. Paired T-tests or Mann-Whitney tests (nonparametric t-test) were used for comparisons between two groups for statistical analysis of in vitro radiation, western blotting quantification and $\mathrm{N}$-acetyl cysteine (NAC) assays. Differences between groups were considered statistically significant at $p<0.05$. Kaplan-Meier survival analysis were calculated using the Log Rank method.

Protein-coding genes were filtered out from the Affymetrix U133 Plus 2 array data leaving a final count of 2858 IncRNAs. Initial identification of IncRNAs in glioblastoma samples from long-term (> 36 months overall survival) versus short-term survivors $(<12$ months overall survival) was carried out using GSE53733 data set based on a prospective cohort of the German Glioma Network [33], excluding the data from patients with intermediate overall survival between 12 and 36 months.

Kaplan-Meier survival curves were generated using both the GSE16011 dataset (Affymetrix U133 Plus 2 array filtered for glioblastoma samples, analyzed using R2 platform (Academic Medical Center (AMC) Amsterdam, the Netherlands)) and the TCGA dataset (Affymetrix Human Exon 1.0 ST array, https://www.cancer.gov/tcga, analyzed using IBM SPSS statistics (version 21 IBM Corporation)). The last quartile was used to define high HOTAIRM1 expression.

Analysis of chromosome 7 gene expression relative to chromosome 7 copy number status was performed by plotting expression fold change according to disomy 7 versus trisomy 7 based on U133 Plus 2 glioblastoma datasets with copy number information $(n=29)$ taken from GSE7696, GSE36245 and GSE43289. In addition TCGA samples from the Affymetrix Human Exon 1.0 ST array were used in separate analyses with samples of undetermined copy number status being removed.

GeneSet Enrichment Analysis was performed using the $t$-value from the paired t-test for both RNA sequencing and proteomics data of the siRNA-mediated knockdown and respective controls. Gene sets were comprised of curated pathways from several databases including GO, Reactome, KEGG (March 242016 version; http://download.baderlab.org/ EM_Genesets/current_release/Human/symbol/) and visualized using Cytoscape (www.cytoscape.org; $p \leq 0.001, q \leq 0.05$, similarity cutoff 0.5 ).

MicroRNA (miRNA) predicted to bind to HOTAIRM1 and either of the 12 candidate proteins that were down-regulated after stable HOTAIRM1 in LN229 glioblastoma cells were identified using the miRanda tool (https:// omictools.com/miranda-tool). Among these miRNAS, we selected those that showed inverse expression relative to HOTAIRM1 in the investigated TCGA data set (https://www.cancer.gov/tcga).

TGM2 promoter methylation status was investigated using a TCGA data set profiled using an illumina $450 \mathrm{~K}$ methylation array (https://www.cancer. gov/tcga). Only a small subset of samples have HOTAIRM1 status since sample overlap was minimal between expression and methylation data.

\section{RESULTS}

High HOTAIRM1 expression is associated with shorter survival of glioblastoma patients

We took advantage of publically available glioma gene expression datasets to identify IncRNAs associated with overall survival of glioblastoma patients. First, we compared IncRNA expression profiles of primary glioblastoma samples from patients with long-term (overall survival $>36$ months) versus short-term (overall survival < 12 months) using the German Glioma Network (GGN) cohort [33]. We found three IncRNAs that were significantly upregulated in the population of short-term survivors. Out of these IncRNAs, HOTAIRM1 was a top candidate based on fold-change expression difference and statistical significance (Fig. 1A, Supplementary Table 1). We validated this observation across the cohort that higher HOTAIRM1 expression also correlated with shorter overall survival (Supplementary Fig. $1 \mathrm{~A})$ and analyzed additional publically available data sets from two independent, non-overlapping patient cohorts published by The Cancer Genome Atlas (TCGA) consortium [34] (https://www. cancer.gov/tcga) and Gravendeel et al. (2009) [35]. Thereby, we confirmed that patients whose tumors show high HOTAIRM1 expression levels, as defined by the upper quartile, demonstrated shorter overall survival (Fig. 1B, C). We confirmed IDH1 mutation as a strong prognostic marker of longer survival in the GGN dataset [33] (Supplementary Fig. 1B) and found that the prognostic value of the HOTAIRM1 expression was independent from IDH1 mutation (Fig. 1D, E) and the MGMT promoter methylation status in this patient cohort (Fig. 1F, Supplementary Fig. 1). Glioblastoma patients whose tumors carried an IDH1 mutation, MGMT promoter methylation and low HOTAIRM1 expression showed the longest overall survival (Fig. 1F). Consistent with these prognostic associations, HOTAIRM1 was recently shown by other investigators to be aberrantly expressed in glioblastoma [21] and associated with shorter survival of glioma patients [21, 24, 26].

Since the majority of glioblastomas display copy number gains of chromosome 7, often due to trisomy 7 [36], we evaluated the expression level of HOTAIRM1 in relation to chromosome 7 copy number status in glioblastomas [37-39]. In addition to the epidermal growth factor receptor (EGFR) gene, i.e., the protooncogene most commonly amplified and overexpressed in IDHwildtype glioblastoma [9], HOTAIRM1 and the protein-coding gene MEOX2 showed consistently increased expression in tumors with chromosome 7 gain when compared to tumors without this copy number increase (Fig. 1G, H). EGFR [40, 41] and MEOX2 [42] overexpression have been implicated before as drivers of glioblastoma growth. HOTAIRM1 was the only IncRNA on chromosome 7 that was significantly upregulated in gliomas with chromosome 7 gain.

\section{HOTAIRM1 knock-down decreases glioblastoma cell viability, invasion, and clonogenicity}

To determine effects of genetic knock-down of HOTAIRM1 in glioma cells, we first performed a transient siRNA-mediated knockdown of HOTAIRM1 in the four established glioblastoma cell lines U251MG, LN-229, LN-18, and T98G (Fig. 2A-D), which showed an intermediate expression level (Supplementary Fig. 2). Efficiency of HOTAIRM 1 knock-down was greater than $80 \%$ in each of the four cell lines (Fig. 2A). HOTAIRM1 knock-down significantly reduced cell viability of these glioma lines by $20-30 \%$ (Fig. 2B). In addition, HOTAIRM 1 knock-down resulted in significant reduction of glioma cell invasiveness by $40-50 \%$ (Fig. 2C, Supplementary Fig. 3) and colony formation capacity by $25-40 \%$ (Fig. 2D).

To further investigate the phenotypic changes caused by HOTAIRM1 knock-down, we generated stable knock-down glioma lines using a lentiviral shRNA approach. Twenty-four hours post transduction, glioma cell lines were selected using either clonal 

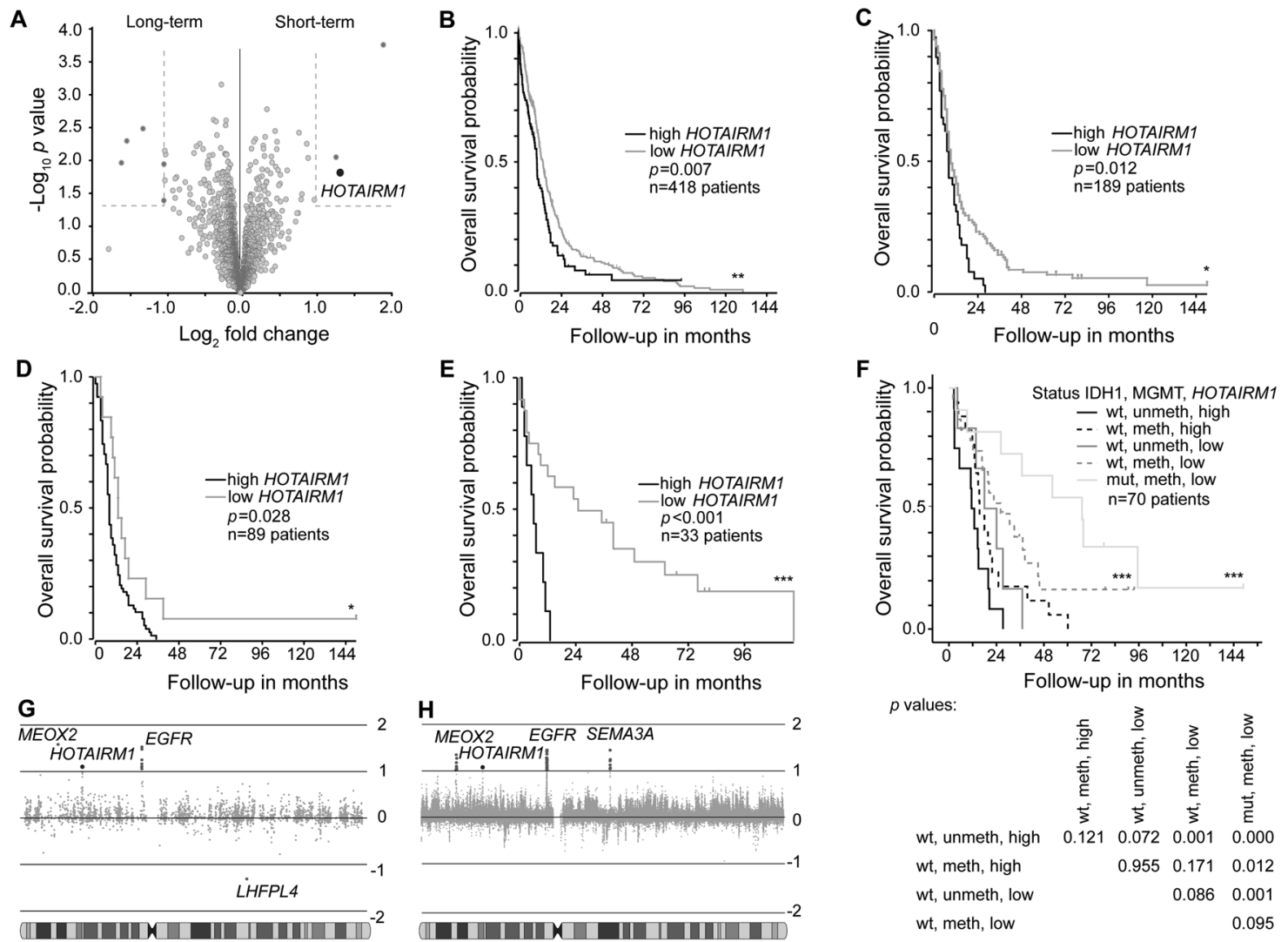

Fig. 1 Prognostic role of HOTAIRM1 expression in glioblastoma patient datasets. A Volcano plot showing differential expression of IncRNAs in glioblastomas from patients with long-term (overall survival $>36$ months) versus short-term (overall survival $<12$ months) survival in the German Glioma Network (GGN) cohort ${ }^{24}$. The black circle highlights HOTAIRM1 while dark gray circles represent other IncRNAs with differential expression between survival groups $( \pm 2$-fold change and $p<0.05)$. Light gray circles indicate IncRNAs that are not significant. B Overall survival plots of glioblastoma patients from TCGA [34] (https://www.cancer.gov/tcga) and C Gravendeel et al. [35] stratified according to high or low HOTAIRM1 expression levels. Cut-off for high HOTAIRM1 was determined by upper quartile and log rank statistics were calculated. D, E Overall survival of glioblastoma patients in the Gravendeel et al. [35] cohort according to HOTAIRM1 expression in IDH1-wildtype (D) and IDH1-mutant glioblastomas (E). F Overall survival of glioblastoma patients in the GGN cohort [33] stratified according to HOTAIRM1 expression, MGMT promoter methylation status, and IDH1 mutation status (wt: wild-type; mut: mutant; meth: methylated; unmeth: unmethylated). The table below the Kaplan-Meier graph lists p-values for the individual subgroups. G, H Expression of genes mapping to chromosome 7 in glioblastomas stratified according to presence or absence of chromosome 7 gain. G Data based on primary glioblastoma [37-39] Affymetrix U133 Plus 2 arrays or (H) TCGA [34] Human Exon 1.0 ST array show HOTAIRM1 as the only IncRNA with significantly increased expression in glioblastomas with chromosome 7 gain in addition to the coding genes EGFR, MEOX2 and SEMA3A. Log rank analysis for Kaplan-Meier survival plots; ${ }^{* *} p<0.001,{ }^{* *} p<0.01,{ }^{*} p<0.05$.

selection with puromycin (LN-229) or selection with blasticidin for pooled populations (LN-18, SF126 and U87MG). Efficient stable knock-down was achieved for HOTAIRM1 in all cell lines (Supplementary Fig. 4A) and the phenotypic characterization was performed as described above for transient models (Supplementary Fig. 4B-D). The stable HOTAIRM1 knock-down cell lines corroborated the phenotype observed in transient models, i.e., cell viability was decreased by $20-40 \%$ (Supplementary Fig. 4B), while cell invasion and colony formation were reduced by $20-70 \%$ (Supplementary Fig. 4C) and 15-70\% (Supplementary Fig. 4D). These findings are in line with recently published data from other groups [21, 24-26].

\section{Proteogenomic analyses reveal evidence for mitochondrial dysfunction upon HOTAIRM1 knock-down}

To characterize molecular mechanisms underlying the observed effects of HOTAIRM1 knock-down on glioma cells in vitro, we performed RNA sequencing and MS-based proteome analyses on three siRNA-mediated HOTAIRM1 knock-down models (U251, LN229 , and T98G) and their respective control-transfected cell lines (Supplementary Tables 2-3). A proteogenomic approach was taken for integrative bioinformatic evaluation of the RNA and protein data sets. First, preranked GeneSet Enrichment Analysis (GSEA) was performed using the t-statistic from the T-test for both the RNA sequencing data (see Supplementary Table 4 and 5 for the lists of positively or negatively enriched genesets identified by RNA sequencing, respectively) and the mass spectrometry data (see Supplementary Table 6 and 7 for the lists of positively or negatively enriched genesets identified by proteome analyses, respectively). The GSEA output was then visualized in cytoscape. The overlapping nodes that were similarly enriched in both datasets contain genesets involved in mRNA processing and translation, as well as mitochondrial translation and mitochondrial membrane function, suggesting that HOTAIRM1 knock-down in glioma cells interferes with mitochondrial and translational functions (Fig. 3A, Supplementary Table 8).

To verify potential mitochondrial dysfunction upon HOTAIRM1 knock-down, we performed immunofluorescent dihydroethidium (HEt) and MitoSOX stainings to measure cytoplasmic and mitochondrial superoxide levels, which is a type of reactive oxygen species (ROS), as an indicator of mitochondrial dysfunction [43]. HOTAIRM1 knock-down cells showed increased ROS levels 

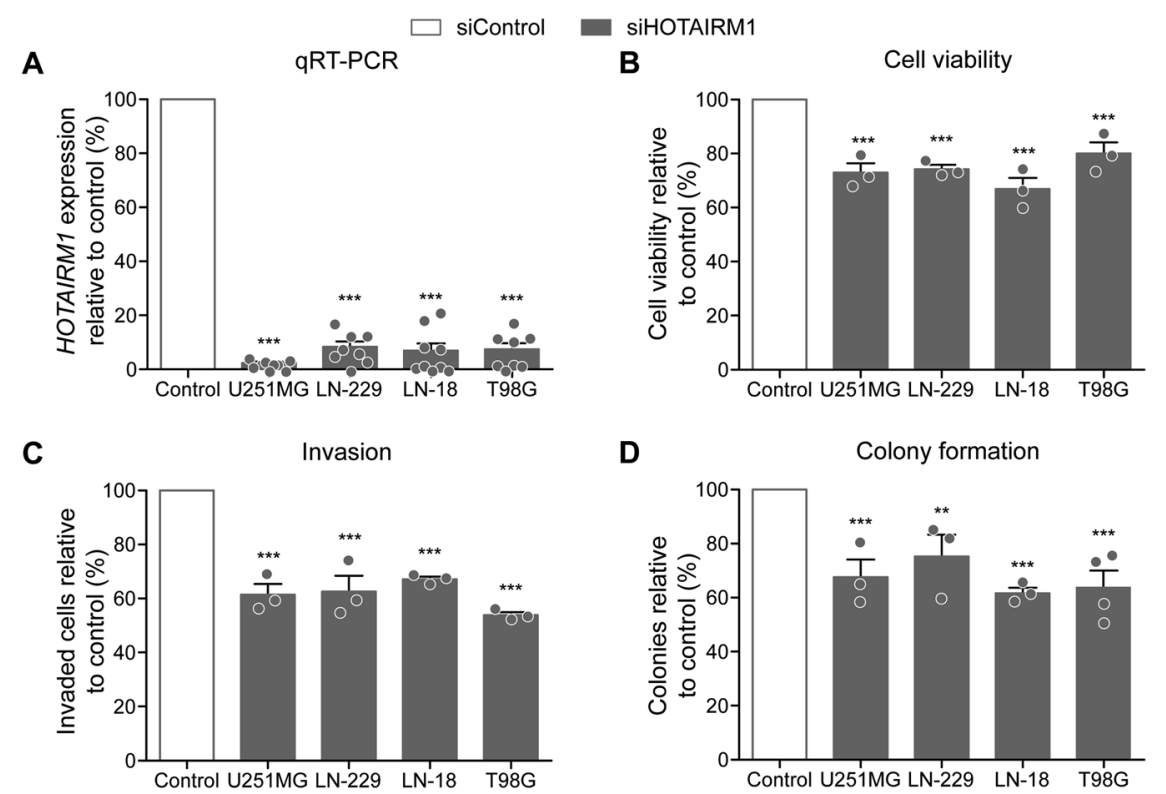

Fig. 2 HOTAIRM1 knock-down decreases oncogenic features in glioblastoma cell lines. siRNA-mediated knock-down was achieved using siPOOLS (siTOOLs Biotech, Planegg, Germany). A qRT-PCR was performed using TaqMan probes against HOTAIRM1 or phosphoglycerate kinase 1 (PGK1) as a housekeeping control gene. Following HOTAIRM1 knock-down, the four investigated glioma cell lines showed reduced cell viability as determined with the CellTiter-Glo assay (B), reduced invasiveness measured in Boyden chamber assays (C), and, finally, decreased clonogenicity as determined by colony formation assays after seeding cells at a density of 500 (U251MG and LN-18) to 1000 (LN-229 and T98G) cells per $10 \mathrm{~cm}$ dish (D). White bars indicate the results of the respective control-transfected cells set to $100 \%$. Filled bars are results obtained with HOTAIRM1 knock-down cells. siControl: cells transfected with non-target siPOOLS; siHOTAIRM1: cells transfected with siPOOLS against HOTAIRM1. Two-way ANOVA was used for statistical analyses; mean $\pm \mathrm{SEM}$, ${ }^{* * *} p<0.001,{ }^{* *} p<0.01 . n=4$ independent experiments for the colony formation assays for T98G cell line, $n=3$ independent experiments per cell line and assay.

compared to control cells (Fig. 3B), a finding in line with the proteogenomic results suggesting deficient mitochondrial function in HOTAIRM1 knock-down glioma cells. Further support for increased ROS levels as relevant driver of cellular effects caused by HOTAIRM 1 knock-down was obtained by treatment of LN-229 and LN-18 glioma cells with $\mathrm{N}$-acetyl cysteine (NAC), a ROS scavenger, which showed that NAC treatment rescued the decrease in colony formation caused by HOTAIRM1 knock-down (Fig. 3C, Supplementary Fig. 5).

\section{HOTAIRM1 knock-down sensitizes glioblastoma cells to radiation in vitro and in vivo}

Since radiation sensitivity has been associated with intracellular ROS levels [44] and since radiotherapy is an essential part of glioblastoma treatment, we investigated whether altered levels of HOTAIRM1 affect radiosensitivity of glioblastoma cells. HOTAIRM1 knock-down and control LN-229, SF126, and LN-18 cells were irradiated with either 2 or $4 \mathrm{~Gy}$ and colony formation capacity was evaluated in relation to non-irradiated cells. After 21 days, surviving colonies revealed a radiation dose-dependent significant decrease in colony formation compared to non-irradiated cells (Fig. 4A-C). HOTAIRM1 knock-down caused reduced colony formation capacity of glioma cells already in non-irradiated cells (Fig. 2D; Supplementary Fig. 4D). However, we observed an additional, dose-dependent decrease in colony formation of HOTAIRM1 knock-down glioma cells after irradiation compared to irradiated control-transfected glioma cells (Fig. 4A-C) which was not caused by an alteration in HOTAIRM1 expression levels (Supplementary Fig. 6).

To validate the radiosensitizing effect of HOTAIRM1 knockdown in vivo, we investigated an orthotopic xenograft glioma model using LN-229 control and HOTAIRM1 knock-down cells. Mice inoculated intracerebrally with LN-229 control or HOTAIRM1 knock-down cells were evaluated for orthotopic tumor growth and survival either with or without single irradiation with $12 \mathrm{~Gy}$ at day 15 post tumor cell transplantation. HOTAIRM1 knock-down did not alter overall survival when mice were not irradiated (Fig. 4D), with control and HOTAIRM1 knock-down LN-229-bearing mice exhibiting median overall survivals of 43 and 44.5 days, respectively. This finding contrasts with a recent study showing reduced in vivo tumor growth of U87MG glioma cells following HOTAIRM1 knockdown [21], which might be related to the different models and experimental conditions. Importantly, however, we found that HOTAIRM1 knock-down LN-229-bearing mice survived significantly longer following radiotherapy when compared to mice transplanted with control-transfected LN-229, as indicated by median overall survivals of 80 versus 38 days, respectively (Fig. 4E).

\section{Transglutaminase 2 (TGM2) is down-regulated upon HOTAIRM1 knock-down in glioblastoma cells}

Mass spectrometry-based proteome analyses using the stable LN-229 HOTAIRM1 knock-down model detected 16 proteins that were upregulated and 12 proteins that were down-regulated upon HOTAIRM1 knock-down (Supplementary Tables 9-11). Transglutaminase 2 (TGM2) was detected as one of the 12 proteins that were down-regulated in HOTAIRM1 knock-down LN-229 cells and also was significantly correlated with HOTAIRM1 in the TCGA glioblastoma patient tissues (Supplementary Fig. 7A). Interestingly, TGM2 has been shown to play a role in mitochondrial function [45] and cancer therapy resistance $[46,47]$. Reduced TGM2 mRNA and protein levels in HOTAIRM1 stable knock-down LN-229, as well as other glioblastoma cell lines (U87MG, LN-18, and SF126) relative to control-transfected cells, were confirmed by RT-qPCR (Fig. 5A) and Western blotting (Fig. 5B-D). We corroborated reduced TGM2 expression upon siRNA-based knockdown of HOTAIRM1 

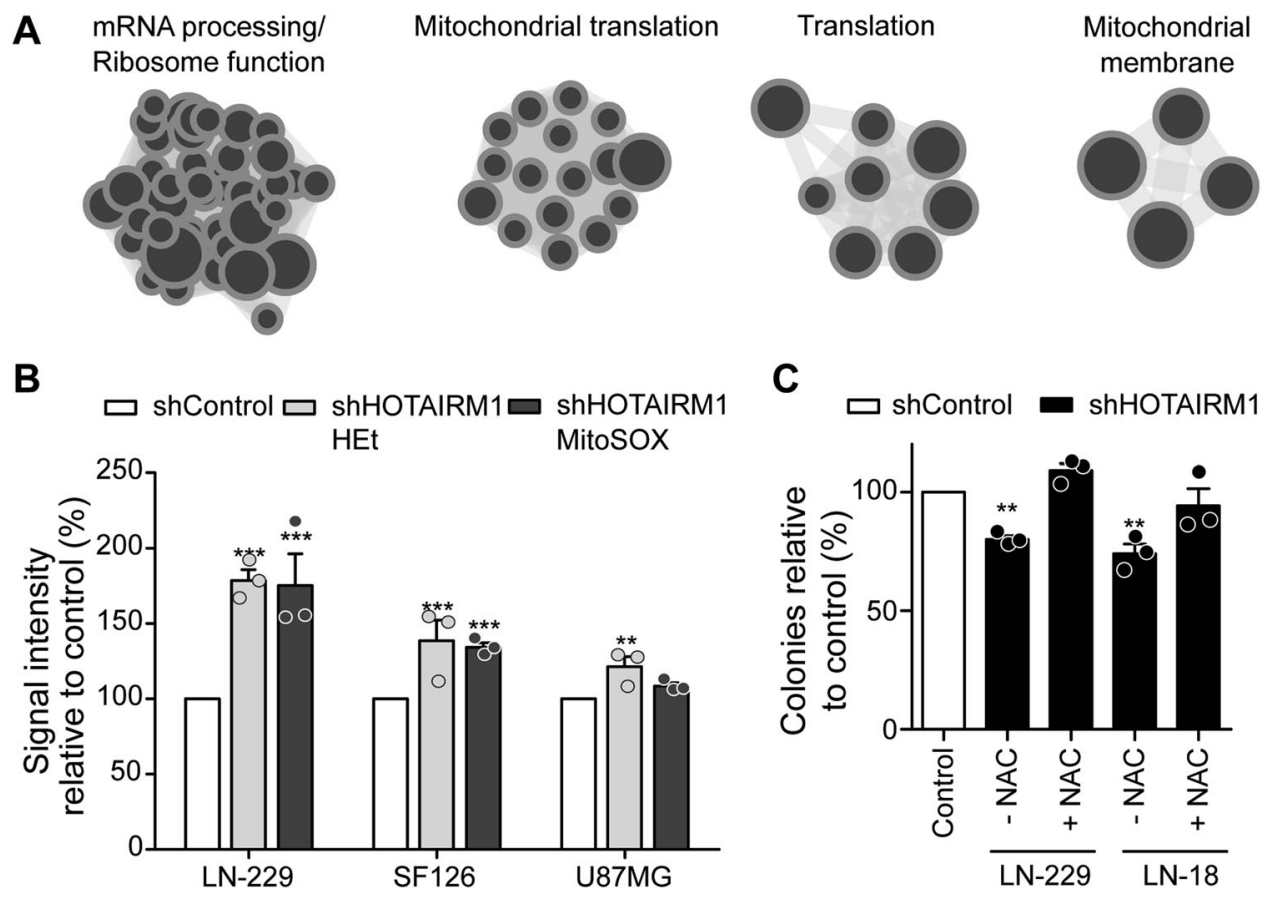

Fig. 3 Knock-down of HOTAIRM1 results in mitochondrial dysfunction and increased reactive oxygen species (ROS). A Merged GSEA of RNA sequencing and proteomics data showing overlapping geneset clusters related to mRNA processing/ribosome function, mitochondrial translation, translation and mitochondrial membrane (see supplementary tables $2-5$ for the list of the genesets). B Quantification of HEt (general superoxide indicator) and MitoSox (mitochondrial superoxide indicator) staining performed on stable LN-229, SF126, and U87MG HOTAIRM1 knock-down cells and respective controls $(n=3)$. Shown are ROS levels normalized to control cells. C Results of colony formation assays 21 days post antioxidant NAC treatment in stable LN-229 and LN-18 HOTAIRM1 knock-down and control cells ( $n=3$ ). Two-way ANOVA was used for statistical analyses; mean $\pm \mathrm{SEM},{ }^{* *} p<0.001,{ }^{* *} p<0.01,{ }^{*} p<0.05$.

in LN-229, T98G, and U87MG, while U251MG showed no detectable expression on protein level of the proposed candidate (Supplementary Fig. 7B).

\section{siRNA-mediated TGM2 knock-down mimics the phenotype of HOTAIRM1 knock-down \\ To investigate functional roles of reduced TGM2 expression in glioblastoma cells, we performed siRNA-mediated knock-down of TGM2 in LN-229 and SF126 glioma cells (Fig. 6A). Similar to HOTAIRM1 knock-down, TGM2 knock-down resulted in signifi- cantly reduced cell viability (Fig. 6B), cell invastion (Fig. 6C), and colony formation (Fig. 6D). Although TGM2 and HOTAIRM1 expression is positively correlated, we did not observe the same survival benefit for HOTAIRM1 (Supplementary Fig. 8A-D), as the strongest prognostic indicator observed was MGMT promoter methylation status (Supplementary Fig. 8E). The in vitro data are in line with studies reporting on tumor-promoting functions of TGM2 in other cancer models $[48,49]$ and suggest regulation of TGM2 by HOTAIRM1 as a putative mechanism driving glioma aggressiveness However, TGM2 expression is not regulated by promoter methylation in patient samples, as analysis of glioblastoma methylation data showed that HOTAIRM1 promoter is unmethy- lated in all samples, independently of high or low HOTAIRM1 expression (Supplementary Fig. 7C).}

\section{Potential regulation of TGM2 by HOTAIRM1 via sponging of hsa-miR-17-5p}

HOTAIRM1 has recently been proposed to function as a sponge for several miRNAs $[24-26,50-54]$ including miR-17-5p [50,53], miR-129-5p, and miR-495-3p [24]. Therefore, we performed in silico analyses to determine putative miRNA binding sites that are shared between HOTAIRM 1 and transcripts of the 12 proteins with significantly reduced expression in HOTAIRM1 knock-down
LN-229 cells. The number of candidate miRNAs with putative binding sites in HOTAIRM1 and any of the 12 candidate gene transcripts was further narrowed down by filtering for those miRNAs whose expression was inversely correlated to the expression of the 12 genes in the TCGA mRNA and miRNA glioblastoma data set (accession: phs000178.v10.p8.c1). Together, these in silico analyses revealed 15 miRNAs targeting HOTAIRM 1 and at least one of the genes of interest (Supplementary Table 12). Interestingly, 14 of the 15 miRNAs had predicted binding sites in both TGM2 and HOTAIRM1 (Supplementary Table 12) which includes members of the miR17-92 cluster, specifically $h s a-m i R-17-5 p$ that has been reported to interact with HOTAIRM1 in gastric and colorectal tumor entities $[50,53]$. We found that low levels of hsa-miR-17-5p expression are associated with shorter survival of glioblastoma patients in the TCGA cohort (Supplementary Fig. 9A) and that expression of hsa-miR-17-5p is inversely correlated with TGM2 expression in this cohort set (Supplementary Fig. 9B), however, not with HOTAIRM1 expression (Supplementary Fig. 9C). To support these data, we performed a miR-17-5p overexpression experiment whereby miR-17-5p mimics were transfected in LN-229 cells. We were able to verify that upon increased miR-17-5p expression levels, TGM2 expression was reduced (Supplementary Fig. 10A). To show that miR-17-5p regulates TGM2 $3^{\prime}$ UTR, we then performed a luciferase assay using either the predicted miR-17$5 p$ binding site of the TGM2 3'UTR (17-5 wt) or a corresponding miR17-5p mutant binding site (17-5 mt) fused to Luciferase. This showed that addition of a miR-17-5p mimic led to a reduction of 17-5 wt, but not of 17-5 mt, driven Luciferase (Supplementary Fig. 10B), altogether indicating that TGM2 transcript is a target of miR-17-5p. Since HOTAIRM1 was shown to bind to miR-17-5p $[50,53]$, our data suggest that HOTAIRM1 regulates TGM2 expression possibly by controlling the availability of miR-17-5p. 

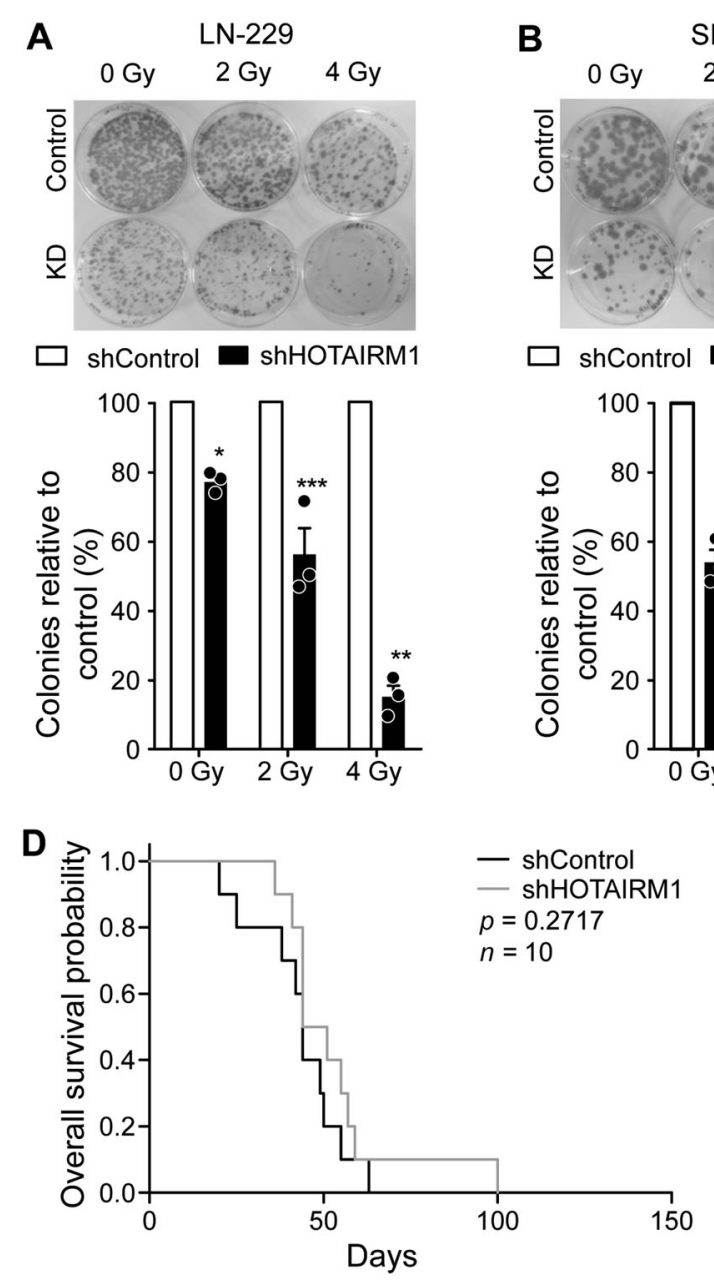
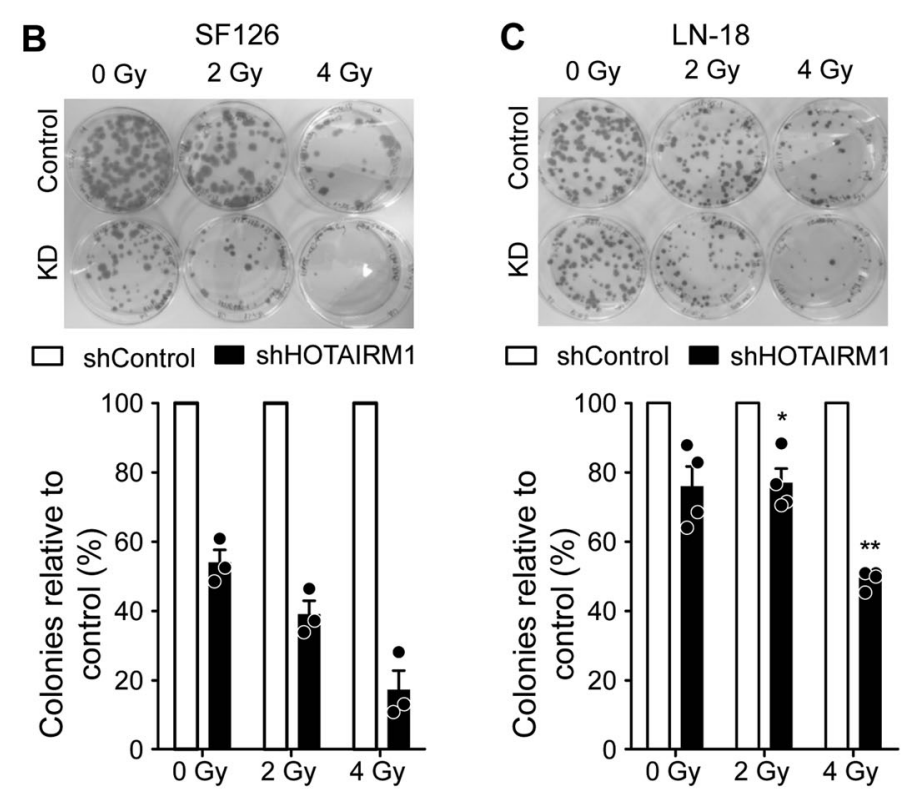

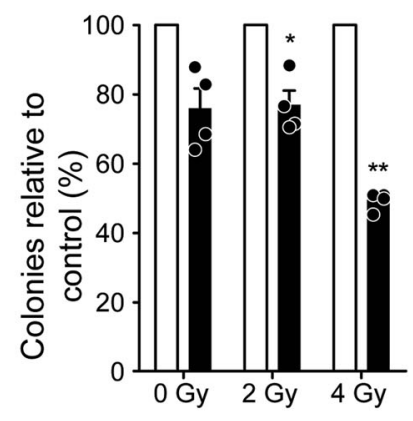

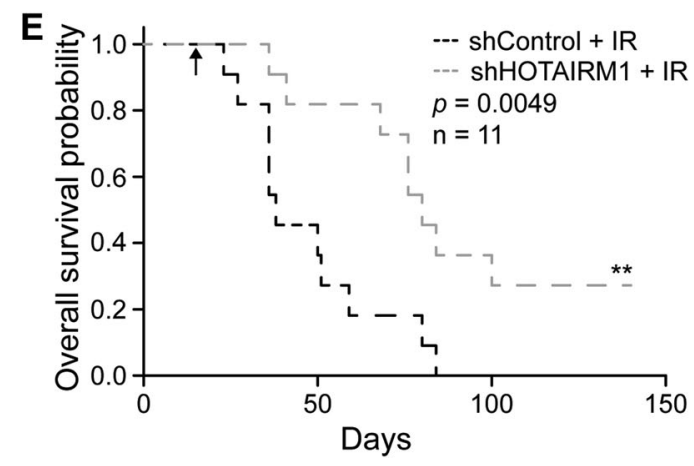

Fig. 4 HOTAIRM1 knock-down sensitizes glioblastoma cells to radiation in vitro and in vivo. A, B, C Representative images and quantification of colony formation assays 21 days post irradiation at indicated doses for stable (A) LN-229, (B) SF126, and (C) LN-18 HOTAIRM1 knock-down (KD) and control cells. Counts were normalized to the corresponding counts in isogenic controls at respective radiation dose (D, E) Kaplan-Meier survival plots of mice harboring either HOTAIRM1 stable knock-down or control transfected LN-229 orthotopic xenografts not treated with radiation (D) and treated with $12 \mathrm{~Gy}$ radiation at day 15 (arrow) (E). Gray and black lines represent HOTAIRM1 knock-down and controls, respectively. shControl: non-target shRNA; shHOTAIRM1: shRNA against HOTAIRM1. Two-way ANOVA was used for colony formation statistical analyses and log rank analysis for Kaplan-Meier survival plots; ${ }^{* * *} p<0.001,{ }^{* *} p<0.01,{ }^{*} p<0.05$.
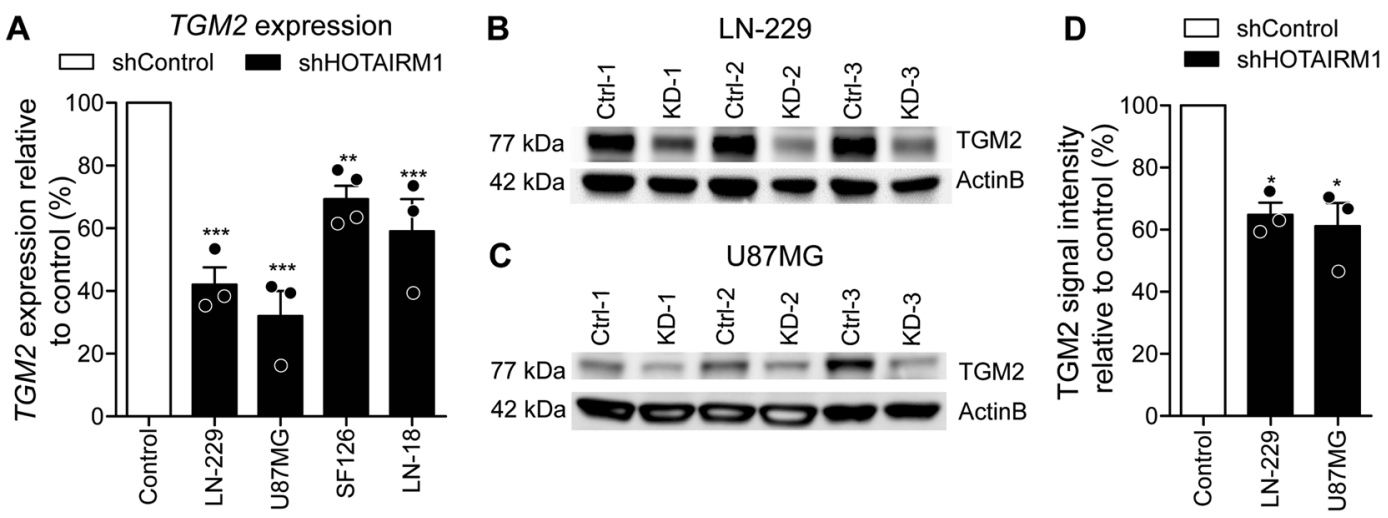

Fig. 5 TGM2 expression is correlated with HOTAIRM1 expression. A qRT-PCR for TGM2 expression was performed in control and stable HOTAIRM1 knock-down LN-229, U87MG, SF126, and LN-18 cell lines. White bar indicates the results of the respective control cells set to $100 \%$. Note that HOTAIRM1 knock-down significantly reduces TGM2 mRNA levels. B-D Western blotting analysis of TGM2 protein expression in control versus stable HOTAIRM1 knock-down LN-229 (B) and U87MG (C) cell lines. Shown are three independent experiments. Beta-actin (ActinB) was used as a loading control. D Quantification of TGM2 protein expression by western blotting analysis in control versus stable HOTAIRM1 knock-down LN-229 and U87MG cell lines. White bar indicates the results of the respective control cells set to $100 \%$. Two-way ANOVA was used for statistical calculation for qRT-PCR and Student's t test was used for statistical analysis. Control: non-target shRNA; KD: shRNA-mediated HOTAIRM1 knock-down; mean \pm SEM, ${ }^{* * *} p<0.001,{ }^{* *} p<0.01,{ }^{*} p<0.05 . n=3$. 

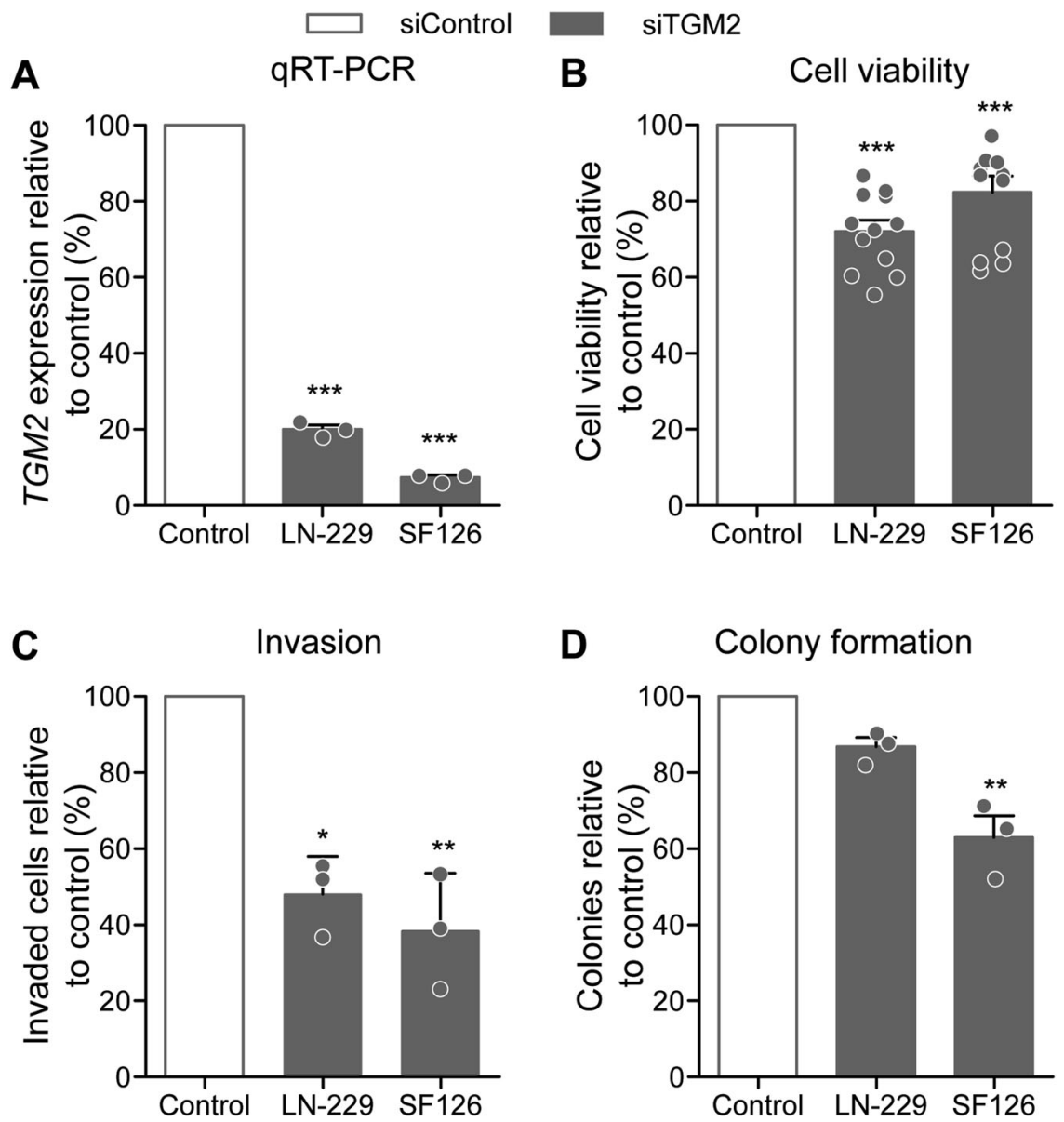

Fig. 6 TGM2 knock-down decreases oncogenic features in glioblastoma cell lines. A qRT-PCR analysis confirms siRNA-mediated knockdown of TGM2 in LN-229 and SF126 glioma cells. Shown are TGM2 mRNA levels normalized to PGK1 mRNA levels relative to control transfected cells set to $100 \%$. Results of determination of cell viability using CellTiter-Glo assays (B), cell invasion using Boyden chamber assays (C), and colony formation propensity using colony formation assays (D) in control versus stable HOTAIRM1 knock-down LN-229 and SF126 cell lines. White bars represent control cells normalized to $100 \%$, filled bars represent TGM2 knock-down cells. Two-way ANOVA was used for statistical analyses; mean \pm SEM, ${ }^{* * *} p<0.001,{ }^{* *} p<0.01 . n=3$.

\section{DISCUSSION}

We have collected compelling evidence from several independent data sets that high HOTAIRM1 expression is linked to clinical aggressiveness and shorter survival of glioblastoma patients and that gene copy number gain is a likely cause of increased HOTAIRM1 expression levels in glioblastoma. After targeting HOTAIRM1 expression in glioblastoma cell lines, the oncogenic potential of these cells was diminished and RNA sequencing and mass spectrometry data suggested impaired mitochondrial function. As it has been shown that cells are more sensitive after temozolomide treatment [24], we decided to focus on the novel finding of mitochondiral disfunction induced upon HOTAIRM1 deficiency. Not only did we validate this finding in vitro, but our data indicate that high expression of HOTAIRM1 supports radioresistance of glioblastoma cells, which in turn may contribute to shorter patient survival as seen in our in vivo model. Since radiation sensitivity has been associated with intracellular ROS levels [44], the modulation of ROS levels by HOTAIRM1 shown in our study suggests HOTAIRM1-mediated reduction of intracellular ROS as a potential mechanism contributing to glioma radioresistance.

To determine additional factors implicated in the HOTAIRM1 mode of action, stable control, and HOTAIRM1 knockdown cell lines have been profiled by proteomic analysis, pointing to TGM2 as a potential mediator of radioresistance. TGM2 is localized in mitochondria as well as in the cytoplasm, endoplasmic reticulum, and plasma membranes [55]. The function of TGM2 in mitochondria is an emerging field [45] and data indicate that TGM2 plays a role in metabolism and mitochondrial respiration [56]. Our data shows that HOTAIRM1 promotes TGM2 expression in glioblastoma cells, which is related to miRNA-mediated mechanisms implicating hsa-miR-17-5p. Our analysis predicted binding sites for hsa-miR-17-5p in both HOTAIRM1 and TGM2 mRNAs. This microRNA, which has been reported to be upregulated by irradiation in glioblastoma [57], has been linked to glioma recurrence [58]. In addition, TGM2 confers radioresistance in different types of cancer cells $[47,59]$. Collectively, these data suggest that HOTAIRM1 may promote glioma growth and therapy resistance by sponging $h s a-m i R-17-5 p$, and thereby increasing TGM2 transcript and protein levels. Thus, in addition to the epigenetic modulation of HOXA1 and the sponging $h s a-$ miR-129-5p and hsa-miR-495-3p, sponging of hsa-miR-17-5p (and potentially other TGM2-binding miRNAs) by HOTAIRM1 may cause increased TGM2 mRNA and protein expression in glioblastoma. However, HOTAIRM1 may also affect other downstream targets as suggested by the absense of TGM2 expresion in U251MG, as the siRNA-based HOTAIRM1 depletion had similar antitumoral effects in this model. Furthermore, we showed HOTAIRM1-mediated TGM2 depletion, which might occur due to hsa-miR-17-5p modulation at the translational level as TGM2 mRNA levels were not affected in T98G, while a reduction in protein levels was detected. 
In summary, we confirm and extend recent data implicating HOTAIRM1 as an oncogenic IncRNA driving tumor growth, therapy resistance, and poor prognosis of glioblastoma. Moreover, our data suggest a novel role for HOTAIRM1 in regulating mitochondrial function and ROS levels in glioblastoma cells by modulating expression of TGM2, potentially by functioning as a miRNA sponge.

\section{REFERENCES}

1. Ostrom QT, Cioffi G, Gittleman H, Patil N, Waite K, Kruchko C, et al. CBTRUS statistical report: primary brain and other central nervous system tumors diagnosed in the United States in 2012-2016. Neuro Oncol. 2019;21:v1-v100.

2. Louis DN, Perry A, Reifenberger G, von Deimling A, Figarella-Branger D, Cavenee WK, et al. The 2016 World Health Organization classification of tumors of the central nervous system: a summary. Acta Neuropathol. 2016;131:803-20.

3. Brat DJ, Aldape K, Colman H, Figrarella-Branger D, Fuller GN, Giannini C, et al. CIMPACT-NOW update 5: recommended grading criteria and terminologies for IDH-mutant astrocytomas. Acta Neuropathol. 2020;139:603-8.

4. Reifenberger G, Wirsching HG, Knobbe-Thomsen CB, Weller M. Advances in the molecular genetics of gliomas - implications for classification and therapy. Nat Rev Clin Oncol. 2017;14:434-52.

5. Stupp R, Mason WP, van den Bent MJ, Weller M, Fisher B, Taphoorn MJ, et al. Radiotherapy plus concomitant and adjuvant temozolomide for glioblastoma. N. Engl J Med. 2005;352:987-96.

6. Stupp R, Taillibert S, Kanner A, Read W, Steinberg D, Lhermitte B, et al. Effect of tumor-treating fields plus maintenance temozolomide vs maintenance temozolomide alone on survival in patients with glioblastoma: a randomized clinical trial. JAMA 2017;318:2306-16.

7. Weller M, van den Bent M, Tonn JC, Stupp R, Preusser M, Cohen-Jonathan-Moyal $\mathrm{E}$, et al. European Association for Neuro-Oncology (EANO) guideline on the diagnosis and treatment of adult astrocytic and oligodendroglial gliomas. Lancet Oncol. 2017; 18:e315-e29.

8. Shah AH, Graham R, Bregy A, Thambuswamy M, Komotar RJ. Recognizing and correcting failures in glioblastoma treatment. Cancer Invest. 2014;32:299-302.

9. Aldape K, Zadeh G, Mansouri S, Reifenberger G, von Deimling A. Glioblastoma: pathology, molecular mechanisms and markers. Acta Neuropathol. 2015; 129:829-48.

10. Hegi ME, Diserens AC, Gorlia T, Hamou MF, de Tribolet N, Weller M, et al. MGMT gene silencing and benefit from temozolomide in glioblastoma. N. Engl J Med. 2005;352:997-1003.

11. Weller M, Tabatabai G, Kastner B, Felsberg J, Steinbach JP, Wick A, et al. MGMT promoter methylation is a strong prognostic biomarker for benefit from doseintensified temozolomide rechallenge in progressive glioblastoma: the DIRECTOR trial. Clin Cancer Res. 2015;21:2057-64.

12. Wick $W$, Weller $M$, van den Bent $M$, Sanson $M$, Weiler $M$, von Deimling $A$, et al. MGMT testing-the challenges for biomarker-based glioma treatment. Nat Rev Neurol. 2014;10:372-85.

13. Le Rhun $E$, Preusser $M$, Roth $P$, Reardon DA, van den Bent $M$, Wen $P$, et al. Molecular targeted therapy of glioblastoma. Cancer Treat Rev. 2019;80:101896.

14. Sigova AA, Mullen AC, Molinie B, Gupta S, Orlando DA, Guenther MG, et al. Divergent transcription of long noncoding RNA/mRNA gene pairs in embryonic stem cells. Proc Natl Acad Sci USA. 2013;110:2876-81.

15. Ayers D, Vandesompele J Influence of microRNAs and Long Non-Coding RNAs in Cancer Chemoresistance. Genes (Basel). 2017;8.

16. Sahu A, Singhal U, Chinnaiyan AM. Long noncoding RNAs in cancer: from function to translation. Trends Cancer. 2015;1:93-109.

17. Heery R, Finn SP, Cuffe S, Gray SG. Long Non-Coding RNAs: Key Regulators of Epithelial-Mesenchymal Transition, Tumour Drug Resistance and Cancer Stem Cells. Cancers (Basel). 2017;9.

18. Zhang X, Lian Z, Padden C, Gerstein MB, Rozowsky J, Snyder M, et al. A myelopoiesis-associated regulatory intergenic noncoding RNA transcript within the human HOXA cluster. Blood 2009;113:2526-34.

19. Chen $Y$, Wu JJ, Lin $X B$, Bao $Y$, Chen ZH, Zhang CR, et al. Differential IncRNA expression profiles in recurrent gliomas compared with primary gliomas identified by microarray analysis. Int J Clin Exp Med 2015;8:5033-43.

20. Zhang X, Sun S, Pu JK, Tsang AC, Lee D, Man VO, et al. Long non-coding RNA expression profiles predict clinical phenotypes in glioma. Neurobiol Dis. 2012;48:1-8.

21. Li Q, Dong C, Cui J, Wang Y, Hong X. Over-expressed IncRNA HOTAIRM1 promotes tumor growth and invasion through up-regulating HOXA1 and sequestering G9a/EZH2/Dnmts away from the HOXA1 gene in glioblastoma multiforme. J Exp Clin Cancer Res. 2018;37:265.
22. Shi T, Guo D, Xu H, Su G, Chen J, Zhao Z, et al. HOTAIRM1, an enhancer IncRNA, promotes glioma proliferation by regulating long-range chromatin interactions within HOXA cluster genes. Mol Biol Rep. 2020;47:2723-33.

23. Xie $P$, Li X, Chen R, Liu Y, Liu D, Liu W, et al. Upregulation of HOTAIRM1 increases migration and invasion by glioblastoma cells. Aging (Albany NY) 2020;13:2348-64.

24. Liang Q, Li X, Guan G, Xu X, Chen C, Cheng P, et al. Long non-coding RNA, HOTAIRM1, promotes glioma malignancy by forming a ceRNA network. Aging (Albany NY). 2019;11.

25. Hao Y, Li X, Chen H, Huo H, Liu Z, Chai E. Over-expression of long noncoding RNA HOTAIRM1 promotes cell proliferation and invasion in human glioblastoma by up-regulating SP1 via sponging miR-137. Neuroreport 2020;31:109-17.

26. Lin YH, Guo L, Yan F, Dou ZQ, Yu Q, Chen G. Long non-coding RNA HOTAIRM1 promotes proliferation and inhibits apoptosis of glioma cells by regulating the miR-873-5p/ZEB2 axis. Chin Med J (Engl) 2020;133:174-82.

27. Livak KJ, Schmittgen TD. Analysis of relative gene expression data using real-time quantitative PCR and the 2 (-Delta Delta C (T)) Method. Methods 2001;25:402-8.

28. Zhang $X$, Weissman SM, Newburger PE. Long intergenic non-coding RNA HOTAIRM1 regulates cell cycle progression during myeloid maturation in NB4 human promyelocytic leukemia cells. RNA Biol. 2014;11:777-87.

29. Rajaraman S, Canjuga D, Ghosh M, Codrea MC, Sieger R, Wedekink F, et al. Measles virus-based treatments trigger a pro-inflammatory cascade and a distinctive immunopeptidome in glioblastoma. Mol Ther Oncolytics 2019;12:147-61.

30. Poschmann G, Seyfarth K, Besong Agbo D, Klafki HW, Rozman J, Wurst W, et al. High-fat diet induced isoform changes of the Parkinson's disease protein DJ-1. J Proteome Res. 2014;13:2339-51.

31. Vizcaino JA, Csordas A, Del-Toro N, Dianes JA, Griss J, Lavidas I, et al. 2016 update of the PRIDE database and its related tools. Nucleic Acids Res. 2016;44:11033.

32. Distelmaier F, Valsecchi F, Liemburg-Apers DC, Lebiedzinska M, Rodenburg RJ, Heil $S$, et al. Mitochondrial dysfunction in primary human fibroblasts triggers an adaptive cell survival program that requires AMPK-alpha. Biochim Biophys Acta 2015;1852:529-40.

33. Reifenberger G, Weber RG, Riehmer V, Kaulich K, Willscher E, Wirth H, et al. Molecular characterization of long-term survivors of glioblastoma using genomeand transcriptome-wide profiling. Int J Cancer 2014;135:1822-31.

34. Cancer Genome Atlas Research N. Comprehensive genomic characterization defines human glioblastoma genes and core pathways. Nature 2008;455:1061-8.

35. Gravendeel LA, Kouwenhoven MC, Gevaert O, de Rooi JJ, Stubbs AP, Duijm JE, et al. Intrinsic gene expression profiles of gliomas are a better predictor of survival than histology. Cancer Res. 2009;69:9065-72.

36. Bigner SH, Mark J, Burger PC, Mahaley MS Jr., Bullard DE, Muhlbaier LH, et al. Specific chromosomal abnormalities in malignant human gliomas. Cancer Res. 1988;48:405-11.

37. Murat A, Migliavacca E, Gorlia T, Lambiv WL, Shay T, Hamou MF, et al. Stem cellrelated "self-renewal" signature and high epidermal growth factor receptor expression associated with resistance to concomitant chemoradiotherapy in glioblastoma. J Clin Oncol. 2008;26:3015-24.

38. Sturm D, Witt $H$, Hovestadt V, Khuong-Quang DA, Jones DT, Konermann C, et al. Hotspot mutations in $\mathrm{H} 3 \mathrm{~F} 3 \mathrm{~A}$ and IDH1 define distinct epigenetic and biological subgroups of glioblastoma. Cancer Cell 2012;22:425-37.

39. Vital AL, Tabernero MD, Castrillo A, Rebelo O, Tao H, Gomes F, et al. Gene expression profiles of human glioblastomas are associated with both tumor cytogenetics and histopathology. Neuro Oncol. 2010;12:991-1003.

40. Chakravarti A, Chakladar A, Delaney MA, Latham DE, Loeffler JS. The epidermal growth factor receptor pathway mediates resistance to sequential administration of radiation and chemotherapy in primary human glioblastoma cells in a RASdependent manner. Cancer Res. 2002;62:4307-15.

41. Mazzoleni S, Politi LS, Pala M, Cominelli M, Franzin A, Sergi Sergi L, et al. Epidermal growth factor receptor expression identifies functionally and molecularly distinct tumor-initiating cells in human glioblastoma multiforme and is required for gliomagenesis. Cancer Res. 2010;70:7500-13.

42. Tachon G, Masliantsev K, Rivet P, Petropoulos C, Godet J, Milin S, et al. Prognostic significance of MEOX2 in gliomas. Mod Pathol. 2019;32:774-86.

43. Bhat AH, Dar KB, Anees S, Zargar MA, Masood A, Sofi MA, et al. Oxidative stress, mitochondrial dysfunction and neurodegenerative diseases; a mechanistic insight. Biomed Pharmacother. 2015;74:101-10.

44. An Z, Yu JR, Park WY. Rosiglitazone enhances radiosensitivity by inhibiting repair of DNA damage in cervical cancer cells. Radiat Environ Biophys. 2017;56:8998.

45. Lai TS, Lin CJ, Wu YT, Wu CJ. Tissue transglutaminase (TG2) and mitochondrial function and dysfunction. Front Biosci (Landmark Ed) 2017;22:1114-37.

46. Li C, Cai J, Ge F, Wang G. TGM2 knockdown reverses cisplatin chemoresistance in osteosarcoma. Int J Mol Med 2018;42:1799-808.

47. Yin J, Oh YT, Kim JY, Kim SS, Choi E, Kim TH, et al. Transglutaminase 2 inhibition reverses mesenchymal transdifferentiation of glioma stem cells by regulating C/ EBPbeta signaling. Cancer Res. 2017;77:4973-84. 
48. Li Z, Xu X, Bai L, Chen W, Lin Y. Epidermal growth factor receptor-mediated tissue transglutaminase overexpression couples acquired tumor necrosis factor-related apoptosis-inducing ligand resistance and migration through c-FLIP and MMP-9 proteins in lung cancer cells. J Biol Chem. 2011;286:21164-72.

49. Hidaka H, Seki N, Yoshino H, Yamasaki T, Yamada $Y$, Nohata $N$, et al. Tumor suppressive microRNA-1285 regulates novel molecular targets: aberrant expression and functional significance in renal cell carcinoma. Oncotarget. 2012;3:44-57.

50. Lu R, Zhao G, Yang Y, Jiang Z, Cai J, Zhang Z, et al. Long noncoding RNA HOTAIRM1 inhibits cell progression by regulating miR-17-5p/ PTEN axis in gastric cancer. J Cell Biochem. 2019;120:4952-65.

51. Zheng M, Liu X, Zhou Q, Liu G. HOTAIRM1 competed endogenously with miR148a to regulate DLGAP1 in head and neck tumor cells. Cancer Med. 2018.

52. Xiao $Y$, Yan X, Yang Y, Ma X. Downregulation of long noncoding RNA HOTAIRM1 variant 1 contributes to osteoarthritis via regulating miR-125b/BMPR2 axis and activating JNK/MAPK/ERK pathway. Biomed Pharmacother. 2019;109:1569-77.

53. Ren T, Hou J, Liu C, Shan F, Xiong X, Qin A, et al. The long non-coding RNA HOTAIRM1 suppresses cell progression via sponging endogenous miR-17-5p/ B-cell translocation gene 3 (BTG3) axis in 5-fluorouracil resistant colorectal cancer cells. Biomed Pharmacother. 2019;117:109171.

54. Chen ZH, Wang WT, Huang W, Fang K, Sun YM, Liu SR, et al. The IncRNA HOTAIRM1 regulates the degradation of PML-RARA oncoprotein and myeloid cell differentiation by enhancing the autophagy pathway. Cell Death Differ. 2017;24:212-24.

55. Bianchi N, Beninati S, Bergamini CM. Spotlight on the transglutaminase 2 gene: a focus on genomic and transcriptional aspects. Biochem J. 2018:475:1643-67.

56. Rodolfo C, Mormone E, Matarrese P, Ciccosanti F, Farrace MG, Garofano E, et al. Tissue transglutaminase is a multifunctional $\mathrm{BH}$-only protein. J Biol Chem. 2004;279:54783-92.

57. Chaudhry MA, Sachdeva H, Omaruddin RA. Radiation-induced micro-RNA modulation in glioblastoma cells differing in DNA-repair pathways. DNA Cell Biol. 2010;29:553-61.

58. Malzkorn B, Wolter M, Liesenberg F, Grzendowski M, Stuhler K, Meyer HE, et al. Identification and functional characterization of microRNAs involved in the malignant progression of gliomas. Brain Pathol. 2010;20:539-50.

59. Arienti C, Tesei A, Carloni S, Ulivi P, Romeo A, Ghigi G, et al. SLUG silencing increases radiosensitivity of melanoma cells in vitro. Cell Oncol (Dordr) 2013;36:131-9.

\section{ACKNOWLEDGEMENTS}

We thank Sarah Göbbels for her technical help and Thomas Hielscher for his support with statistical analyzes. This project was supported by a joint grant from the Deutsche Forschungsgemeinschaft (German Research Foundation) and the Swiss National Science Foundation to M.R., G.R., P.R., and M.W. (RE2857/2-1, RE938/4-1; SNF 310030E_170717). This work was also partly supported by the Deutsche Forschungsgemeinschaft (German Research Foundation) [RE2857/4-1, SCHE 656/2-1 (KFO 337)]. J.T.S. is supported by the German Cancer Consortium (DKTK), the Deutsche Forschungsgemeinschaft (DFG) grant SI1549/3-1 (DFG/GRC-CRU337) and SI1549/4-1, and the German Cancer Aid (Grant no. 70112505; PIPAC consortium).

\section{AUTHOR CONTRIBUTIONS}

U.A., J.B., M.S., M.M., M.L., A.S., M.W. J.K.M.L., D.P., F.S., A.F. and F.D.M. performed the experiments. U.A. and D.P. analyzed the data and wrote the paper. D.P. performed all data analyses. J.B., G.R. and G.L. edited the manuscript. M.T.A., N.Q., A.M.W., L.B., V.M., S.S., C.H., L.K., A.H., C.M., C.B.K.T., A.S., U.F., K.S., S.F., J.T.S., F.D. and A.B. revised the article critically for important intellectual content. G.R., P.R., M.W. and M.R. provided guidance on experimental technology. M.R. is the corresponding author and designed the research. All authors read and approved the final manuscript.

\section{FUNDING}

Open Access funding enabled and organized by Projekt DEAL.

\section{ETHICS STATEMENT}

All animal experiments were performed in accordance with the guidelines of Swiss federal law on animal protection. No human subjects were part of this study.

\section{COMPETING INTERESTS}

G.R. has received honoraria for advisory boards from Abbvie. M.W. has received research grants from Abbvie, Adastra, Bristol Meyer Squibb (BMS), Dracen, Merck, Sharp \& Dohme (MSD), Merck (EMD), Novocure, Piqur and Roche, and honoraria for lectures or advisory board participation or consulting from Abbvie, Basilea, Bristo Meyer Squibb (BMS), Celgene, Merck, Sharp \& Dohme (MSD), Merck (EMD), Novocure, Orbus, Roche and Tocagen. P.R. has received honoraria for advisory board participation and lectures from Bristol-Myers Squibb, Covagen, Debiopharm, Medac, Merck, MSD, Novocure, QED, Roche and Virometix. C.M. is supported by the Heinz Ansmann foundation. JTS reports the following disclosures: Bristol-Myers Sqibb, Celgene, Roche (Research Funding); AstraZeneca, Bristol-Myers Squibb, Celgene, Immunocore, Novartis, Roche, Shire (Consulting or advisory role); AstraZeneca, Aurikamed, Baxalta, Bristol Myers Squibb, Celgene, Falk Foundation, iomedico, Immunocore, Novartis, Roche, Shire (honoraria); minor equity in iTheranostics and Pharma15 (<3\%) and member of the Board of Directors for Pharma15, all outside the submitted work. The other authors report no conflict of interest.

\section{ADDITIONAL INFORMATION}

Supplementary information The online version contains supplementary material available at https://doi.org/10.1038/s41419-021-04146-0.

Correspondence and requests for materials should be addressed to Marc Remke.

Reprints and permission information is available at http://www.nature.com/ reprints

Publisher's note Springer Nature remains neutral with regard to jurisdictional claims in published maps and institutional affiliations.

\begin{abstract}
Open Access This article is licensed under a Creative Commons Attribution 4.0 International License, which permits use, sharing, adaptation, distribution and reproduction in any medium or format, as long as you give appropriate credit to the original author(s) and the source, provide a link to the Creative Commons license, and indicate if changes were made. The images or other third party material in this article are included in the article's Creative Commons license, unless indicated otherwise in a credit line to the material. If material is not included in the article's Creative Commons license and your intended use is not permitted by statutory regulation or exceeds the permitted use, you will need to obtain permission directly from the copyright holder. To view a copy of this license, visit http://creativecommons. org/licenses/by/4.0/.
\end{abstract}

(c) The Author(s) 2021 